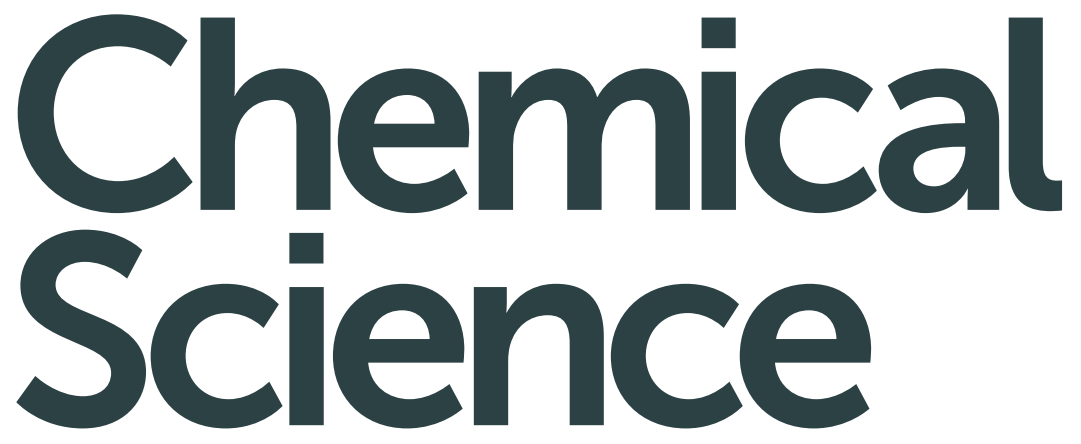

www.rsc.org/chemicalscience

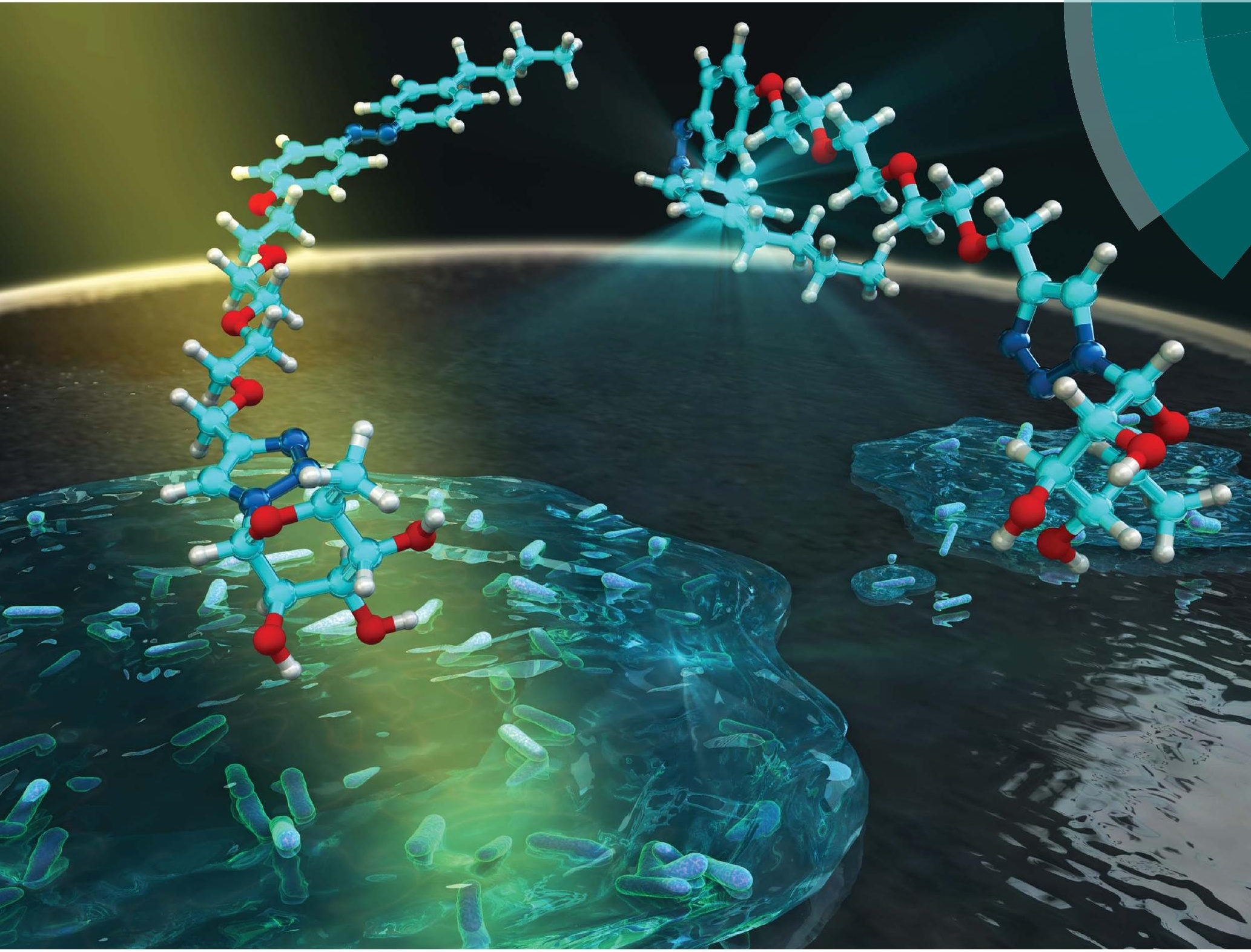

ISSN 2041-6539

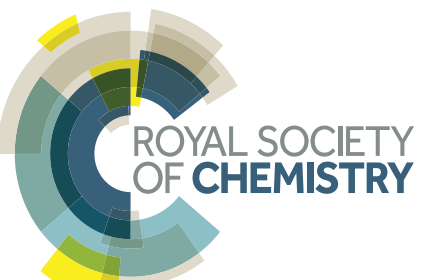

EDGE ARTICLE

Vipul Bansal, Brendan L. Wilkinson et al.

Photomodulation of bacterial growth and biofilm formation using carbohydrate-based surfactants

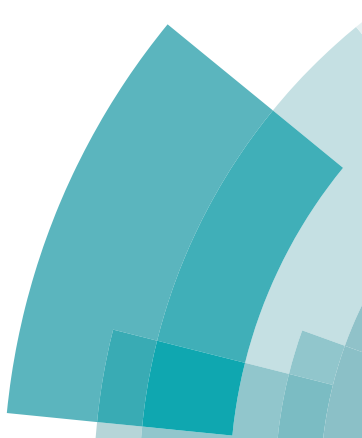




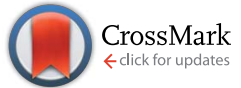

Cite this: Chem. Sci., 2016, 7, 6628

\title{
Photomodulation of bacterial growth and biofilm formation using carbohydrate-based surfactants $\dagger$
}

\author{
Yingxue Hu, $t^{\mathrm{a}}$ Wenyue Zou,,$^{\mathrm{b}}$ Villy Julita, ${ }^{a}$ Rajesh Ramanathan, ${ }^{\mathrm{b}}$ Rico F. Tabor, ${ }^{a}$ \\ Reece Nixon-Luke, ${ }^{c}$ Gary Bryant, ${ }^{c}$ Vipul Bansal ${ }^{\star b}$ and Brendan L. Wilkinson*d
}

Naturally occurring and synthetic carbohydrate amphiphiles have emerged as a promising class of antimicrobial and antiadhesive agents that act through a number of dynamic and often poorly understood mechanisms. In this paper, we provide the first report on the application of azobenzene trans-cis photoisomerization for effecting spatial and temporal control over bacterial growth and biofilm formation using carbohydrate-based surfactants. Photocontrollable surface tension studies and small angle neutron scattering (SANS) revealed the diverse geometries and dimensions of self-assemblies (micelles) made possible through variation of the head group and UV-visible light irradiation. Using these light-addressable amphiphiles, we demonstrate optical control over the antibacterial activity and formation of biofilms against multi-drug resistant (MDR) Pseudomonas aeruginosa, methicillin-resistant Staphylococcus aureus (MRSA) and Gram-negative Escherichia coli. To probe the mechanism of bioactivity further, we evaluated the impact of trans-cis photoisomerization in these surfactants on bacterial motility and revealed photomodulated enhancement in swarming motility in $P$. aeruginosa. These light-responsive amphiphiles should attract significant interest as a new class of antibacterial agents and as investigational tools for probing the complex mechanisms underpinning bacterial adhesion and biofilm formation.

Received 8th July 2016

Accepted 3rd August 2016

DOI: $10.1039 /$ c6sc03020c

www.rsc.org/chemicalscience

\section{Introduction}

Antimicrobial drug resistance represents a global health emergency and there is now an urgent and unmet need for new clinical agents and preventative strategies that possess unconventional modes of action. ${ }^{1}$ This problem is exacerbated by the ability of many pathogenic bacteria to form matrixenclosed communities that are immobilized on biotic and abiotic surfaces, or biofilms, which confer enhanced resistance toward host immune responses and antibiotic treatments. ${ }^{2}$ Naturally occurring biosurfactants are a structurally diverse class of amphiphilic compound with promising antimicrobial and anti-biofilm activity. In particular, microbial glycolipids and their synthetic analogues have attracted considerable interest as selective antibacterial agents and as

\footnotetext{
${ }^{a}$ School of Chemistry, Monash University, Victoria 3800, Australia

${ }^{b}$ Ian Potter NanoBioSensing Facility, NanoBiotechnology Research Laboratory, School of Science, RMIT University, Victoria 3000, Australia. E-mail: vipul.bansal@rmit.edu. $a u$

${ }^{c}$ Centre for Molecular and Nanoscale Physics, School of Science, RMIT University, Victoria 3000, Australia

${ }^{d}$ School of Science and Technology, The University of New England, New South Wales 2351, Australia.E-mail: Brendan.wilkinson@une.edu.au

$\dagger$ Electronic supplementary information (ESI) available: Experimental procedures, supplementary tables, figures and spectra. See DOI: 10.1039/c6sc03020c

\$ Equal contributing authors.
}

mechanistic tools for expanding our understanding of bacterial physiology and biofilm formation. ${ }^{3,4}$ In many cases, this bioactivity can be attributed to the ability of these molecules to lower interfacial tension, thereby mediating bacterial motility, cellular and protein adhesion, signalling and communication, $\mathrm{pH}$ regulation, nutrient uptake, and degradation of harmful metabolites. ${ }^{3-6}$ These multifaceted responses are dependent on the bacterial strain and surfactant used, and our current understanding is limited to a handful of well-characterized systems. Access to chemical tools with tuneable properties that could selectively influence bacterial growth and biofilm formation offers new opportunities to develop novel therapies for biofilm-associated diseases, and to study the mechanisms governing bacterial adhesion and biofilm formation.

In recent years there has been considerable interest in light as an external stimulus for effecting spatial and temporal control over the conformational dynamics of biomolecules ${ }^{7}$ and the biological activity of small molecules. ${ }^{8}$ Toward this goal, the well-studied azobenzene chromophore has been actively pursued, owing to the facile and reproducible trans-cis photoisomerization, and the ease of molecular synthesis for enabling new light-addressable materials with tailored photoswitching wavelengths and potential in vivo applications., ${ }^{\mathbf{9 1 0}}$ In parallel with these developments, azobenzene photochromism has been widely reported as a means for modulating 
A

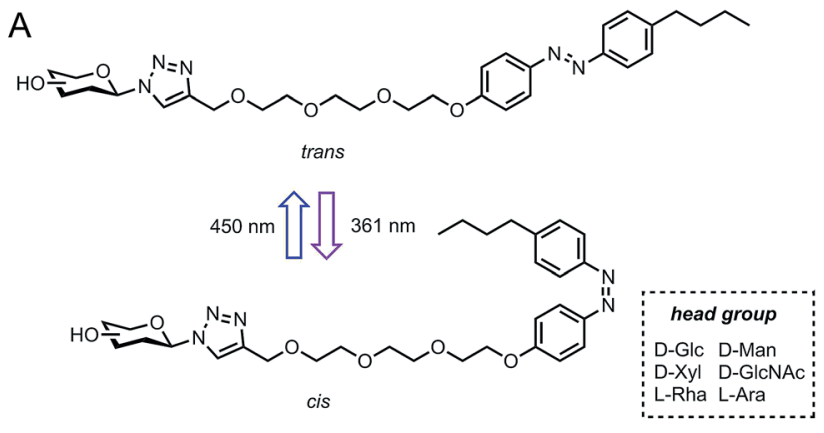

B

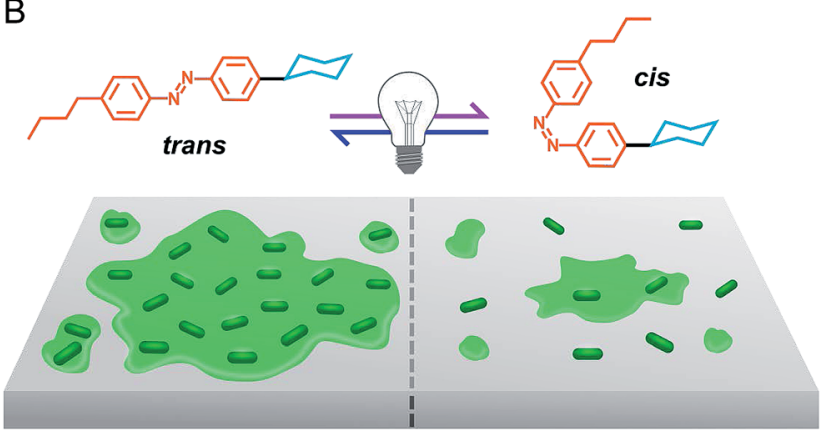

Fig. 1 (A) Photoswitchable carbohydrate-based surfactants and (B) cartoon representation of the photomodulation of biofilm growth.

carbohydrate multivalency and lectin binding, ${ }^{11}$ and for controlling supramolecular self-assembly ${ }^{12}$ and host-guest interactions. ${ }^{13}$ We have previously demonstrated the photocontrollable self-assembly and interfacial activity of carbohydrate-based surfactants and fluorosurfactants. ${ }^{14}$ Throughout the course of these studies, we demonstrated the ability to modulate the interfacial activity and aggregation properties of these amphiphiles through changes in head group geometry, size and polarity, as well as the isomeric state of the tail group..$^{15}$ However, the impact of azobenzene trans-cis photoisomerization within carbohydrate-based surfactants on the ability of bacteria to survive and to form active biofilms remains to be verified.

Herein, we report the photocontrollable antibacterial and biofilm modulatory activity of a panel of carbohydrate-based surfactants (Fig. 1). Surfactants incorporated variable monosaccharide head groups, including D-glucose (AzoGlc), ${ }^{14} \mathrm{D}$-xylose (AzoXyl), L-rhamnose (AzoRha), D-mannose (AzoMan), $N$-acetyl glucosamine (AzoGlcNAc), and L-arabinopyranose (AzoAra), which were tethered to a hydrophobic $n$-butylazobenzene tail group (Fig. 2). Monosaccharide head groups were selected based the natural occurrence and intriguing biological activity of glycolipids expressing these structures, and the important roles carbohydrates play in bacterial adhesion processes. Through reversible photocontrol over self-assembly and interfacial activity, these surfactants were assessed as modulators of bacterial growth and biofilm formation against methicillin resistant Staphylococcus aureus ATCC1698 (MRSA), Escherichia coli DH5 $\alpha$ and a multi-drug resistant strain of Pseudomonas aeruginosa MDR283/1-6.
Surfactants

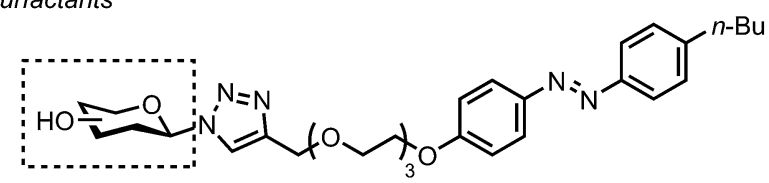<smiles>CC1OC(CO)(CO)C(O)C(O)C1O</smiles><smiles>CC1CC(O)C(O)OCC1O</smiles>

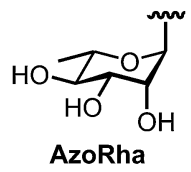<smiles>CC(C)C1C(O)C(CO)C(O)C1CO</smiles>

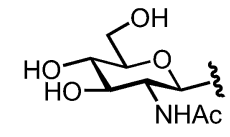<smiles>CC1OCC(O)OC(C)C1O</smiles>

AzoMan
AzoAra<smiles>[R]c1ccc(N=Nc2ccc(CCCC)cc2)cc1</smiles>

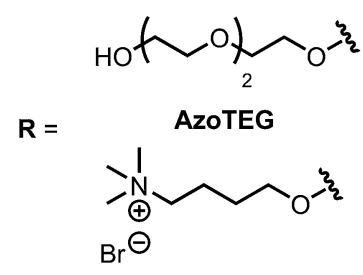

AzoTAB

Fig. 2 Photoswitchable carbohydrate-based surfactants and control compounds used in this study.

\section{Results and discussion}

\section{Surfactant synthesis and characterisation}

Our investigation commenced with the modular synthesis and physical characterisation of a panel of photoswitchable carbohydrate-based surfactants (see ESI for details $\dagger$ ). To this end, known glycosyl azides were united with a $n$-butylazobenzene tail fragment using the well-established $\mathrm{Cu}(\mathrm{I})$-catalysed azide-alkyne cycloaddition reaction (CuAAc). ${ }^{14,16}$ Surfactants were isolated in acceptable yields over two steps following purification by reversed phase, preparative HPLC. In addition to non-ionic carbohydrate photosurfactants, we also targeted a non-surface active fragment lacking the sugar head group (AzoTEG) and a known cationic photosurfactant (AzoTAB) $)^{17}$ as control compounds.

Having acquired surfactants, we assessed the photocontrollable surface activity and self-assembly properties using pendant drop tensiometry and small angle neutron scattering (SANS), respectively. To affect trans-cis photoisomerization, compounds were irradiated at $361 \mathrm{~nm}$ for $15 \mathrm{~min}$ and the critical micellar concentration (CMC) measured using pendant drop tensiometry, although one minute was found to be sufficient to achieve near complete trans-cis photoisomerization. Photoisomerization was also observed using UV-vis spectroscopy as determined by a diminished intensity of the peak at $350 \mathrm{~nm}$ corresponding to the $\pi-\pi^{*}$ transition and an increase in intensity of the peak at $440 \mathrm{~nm}$ corresponding to the $n-\pi^{*}$ 
transition. The ratio of cis and trans isomers in either photostationary state (PSS) was estimated by integrating selected signals in the ${ }^{1} \mathrm{H}$ NMR spectrum, before and after UV photoirradiation (ESI, Fig. S1 $\dagger$ ). In the trans dominated PSS, $<10 \%$ of molecules existed in the cis isomeric form. Following UV irradiation, approximately $75 \%$ of molecules existed in the cis isomeric form (cis dominated PSS). ${ }^{\mathbf{1 8 1 9}}$ To investigate potential photodegradation of surfactants during photoswitching, the ${ }^{1} \mathrm{H}$ NMR spectra of the trans isomer was recorded both prior to UV photoirradiation, and following visible light irradiation $(430 \mathrm{~nm})$ of the photo-excited cis isomer (ESI, Fig. S2 $\dagger$ ).

Since our antibacterial assays occurred in dark-adapted conditions at $37{ }^{\circ} \mathrm{C}$, we were interested in monitoring the thermal relaxation of the cis isomer to the more stable trans isomer in bacterial growth media over a $24 \mathrm{~h}$ time period. The cis isomers showed a half-life of over 10 hours in water as well as in two bacterial growth media at $37{ }^{\circ} \mathrm{C}$ (ESI, Table S1 and Fig. S4-S7†). Considering the typical bacterial doubling time of 0.5-2 $\mathrm{h}$, this would offer ample opportunity for cis isomers to exert a biological response. An increase in the CMC was accompanied by trans-cis photoisomerization, which was attributed to the increased polarity and altered molecular geometry of the cis isomer relative to the more hydrophobic and planar trans isomer (Table 1). ${ }^{20}$ However, in some cases the magnitude of this change was significant, in particular that of AzoGlcNAc incorporating an $\mathrm{N}$-acetyl glucosamine head group. In contrast, virtually no difference in the CMC was observed between the trans $(0.33 \mathrm{mM})$ and cis $(0.34 \mathrm{mM})$ dominated PSS of Azoxyl, further highlighting the effect of subtle head group configuration and polarity on the interfacial activity of this class of photosurfactants.

\section{Characterisation of self-assemblies}

Small angle neutron scattering (SANS) is a powerful experimental technique that is frequently employed to deduce the morphology and size of nanostructures and self-assemblies in solution. ${ }^{\mathbf{1 4 2 1}}$ SANS measurements were performed to determine the post-CMC aggregation structures (micelles) formed by the photoswitchable surfactants, which provided information on the number and topology of carbohydrates exposed on the micelle surface. We postulated that this information could

Table 1 Photocontrollable surface and aggregation properties of carbohydrate-based photosurfactants ${ }^{a}$

\begin{tabular}{lllll}
\hline Surfactant & E/C & $N_{\text {agg }}$ & $A_{\text {hg }}$ & CMC (CMC \\
\hline AzoGlc $^{17}$ & $\mathrm{E}$ & 440 & 0.69 & $0.31(0.59)$ \\
AzoXyl $_{\text {AzoRha }}$ & $\mathrm{C}$ & $\mathrm{NA}$ & $\mathrm{NA}$ & $0.33(0.34)$ \\
AzoMan & $\mathrm{C}$ & 622 & 0.79 & $0.11(0.42)$ \\
AzoGlcNAc & $\mathrm{E}$ & 141 & 0.84 & $0.13(0.49)$ \\
AzoAra & $\mathrm{E}$ & 178 & 1.02 & $0.11(1.22)$ \\
& $\mathrm{E}$ & 89 & 1.12 & $0.33(0.88)$
\end{tabular}

${ }^{a}$ Aggregation number $\left(N_{\mathrm{agg}}\right)$, area per head group $\left(A_{\mathrm{hg}}\right)\left[\mathrm{nm}^{2}\right]$ and critical micelle concentration (CMC) in ambient and UV-irradiated conditions $[\mathrm{mM}] .{ }^{b} \mathrm{E}=$ ellipsoid, $\mathrm{C}=$ cylindrical. For Azoxyl, long worm-like micelles were obtained with a length of $>100 \mathrm{~nm}$. be used to probe the potential relationship between the aggregation properties of these amphiphiles and the ability to modulate bacterial growth and biofilm formation. For each surfactant, spectra were acquired in $\mathrm{D}_{2} \mathrm{O}$ in the trans-dominated PSS at a concentration well above the CMC. As revealed from the SANS data (Table 1 and Fig. 3), a wide range of aggregate morphologies and sizes are made possible through variation of the carbohydrate head group, which is in agreement with our previous studies. ${ }^{\mathbf{1 4 , 1 6}}$ Indeed, micelles ranged from small ellipsoids, as in AzoMan and AzoAra, to extremely long, flexible cylindrical (worm-like) micelles arising from Azoxyl (length > $100 \mathrm{~nm}$, persistence length $36 \mathrm{~nm}$ ). This extraordinary variety of self-assembled structures indicates that the aggregation properties of carbohydrate amphiphiles are extremely sensitive to the head-group employed, offering considerable geometric information on preferred packing and interfacial curvature.

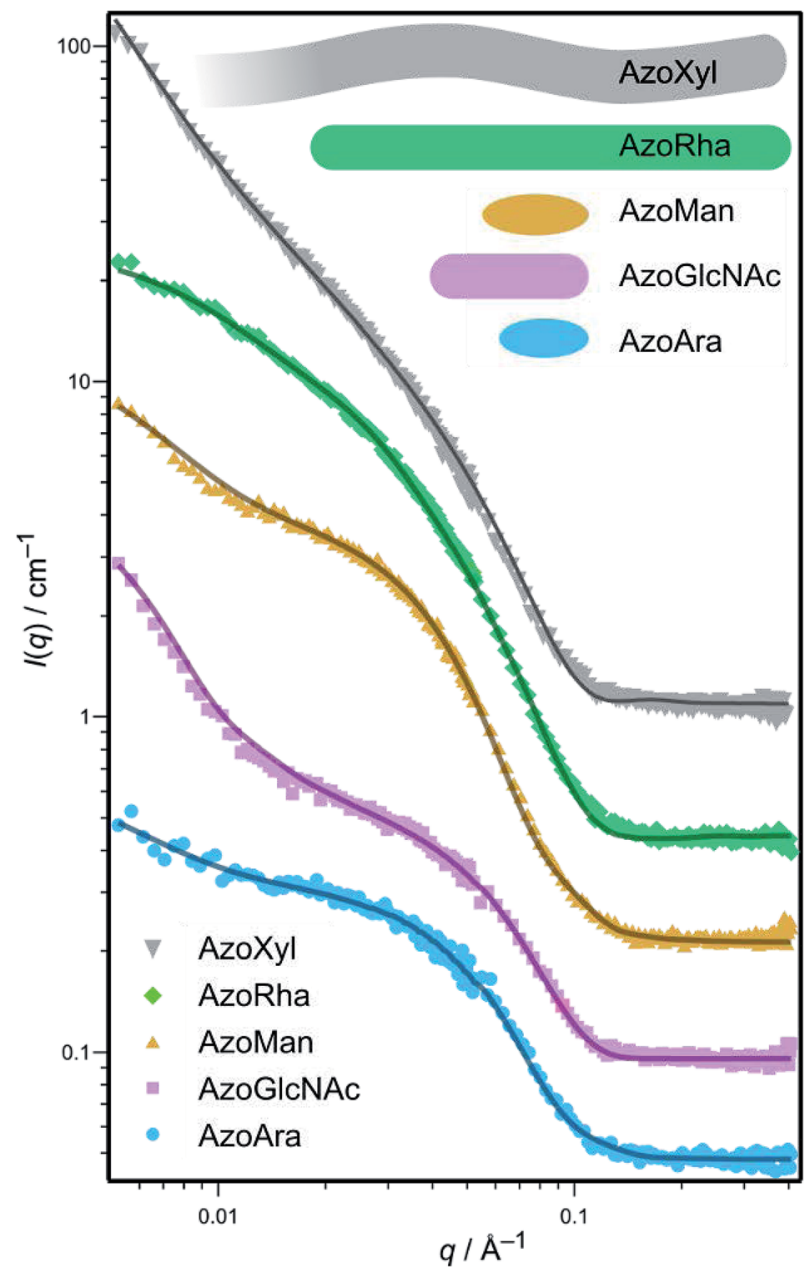

Fig. 3 Small angle neutron scattering (SANS) spectrum of carbohydrate-based surfactants in $\mathrm{D}_{2} \mathrm{O}$ at $4 \mathrm{mM}$ concentration (symbols) and theoretical fits (solid lines) for selected surfactant micelles. Inset top: meridional sections of the fitted geometries from SANS of the surfactants. The SANS spectra for AzoGlc has been reported previously. ${ }^{17}$ 


\section{Photocontrollable antibacterial studies}

We next evaluated the photocontrollable antibacterial activity of photoswitchable surfactants, non-surface active tail fragment AzoTEG and the cationic surfactant AzoTAB against Gramnegative E. coli DH5 $\alpha$, Gram-negative P. aeruginosa, and Grampositive $S$. aureus. Compounds were screened for antibacterial activity using an $\mathrm{OD}_{600}$ bacterial growth assay (see ESI for experimental procedure and data $\dagger$ ). ${ }^{22}$ To obtain cis photoisomers for antibacterial studies, the native compounds were irradiated with UV light for $5 \mathrm{~min}$ prior to incubation with bacteria. Following a dark-adapted $24 \mathrm{~h}$ incubation period, the optical density of the wells was measured at $600 \mathrm{~nm}$. AzoTEG displayed moderate inhibitory potency against $S$. aureus (MIC $125 \mu \mathrm{g} \mathrm{mL}{ }^{-1}$ ), and limited-to-no inhibition of $P$. aeruginosa $\left(\mathrm{MIC}>1000 \mu \mathrm{g} \mathrm{mL}^{-1}\right)$ and E. coli $\left(\mathrm{MIC}>2000 \mu \mathrm{g} \mathrm{mL}^{-1}\right.$ ) in the trans PSS. Following photoisomerization to the cis isomer, a further increase in inhibitory potency against $S$. aureus was observed $\left(31 \mu \mathrm{g} \mathrm{mL} \mathrm{m}^{-1}\right)$, while no change in activity against $E$. coli and $P$. aeruginos $a$ was noted. The enhanced antibacterial activity of the cis isomer of this compound corroborates well with a previous study on photoswitchable quinolone antibiotics. ${ }^{8 c}$ In contrast to the non-surface active fragment, the cationic photosurfactant AzoTAB displayed potent antibacterial activity in the trans PSS against E. coli (MIC $12.5 \mu \mathrm{g} \mathrm{mL} \mathrm{m}^{-1}$ ), S. aureus (MIC $1.6 \mu \mathrm{g} \mathrm{mL}^{-1}$ ), and P. aeruginosa (MIC $31.2 \mu \mathrm{g} \mathrm{mL}^{-1}$ ). While no photomodulation of this activity was observed against $E$. coli and $S$. aureus, the cis PSS of AzoTAB became less toxic against $P$. aeruginosa (MIC $125 \mu \mathrm{g} \mathrm{mL}^{-1}$ ). The high toxicity of the cationic surfactant against $E$. coli and $S$. aureus indicates a non-selective mode of action through cell wall damage and lysis, ${ }^{23}$ whereas its lower toxicity against $P$. aeruginosa allows its activity to be photomodulated, possibly via reduction in the interfacial activity due to the enrichment of the cis isomer. ${ }^{17}$

The carbohydrate-based surfactants exhibited bacteria- and photoisomer-specific, dose-dependent inhibition of growth against both $S$. aureus (ESI, Fig. S7†) and E. coli (ESI, Fig. S8 $\dagger$ ), whereas they generally promoted growth of $P$. aeruginosa (ESI, Fig. S9†). Representative data for AzoMan is shown in Fig. 4. While at lower concentrations, surfactants showed nearly similar activities in the trans- and cis-dominated PSS against all three bacteria, the bactericidal activity could be modulated following photoisomerization at concentrations approaching and above the CMC. In the case of $S$. aureus, the photoexcited cis
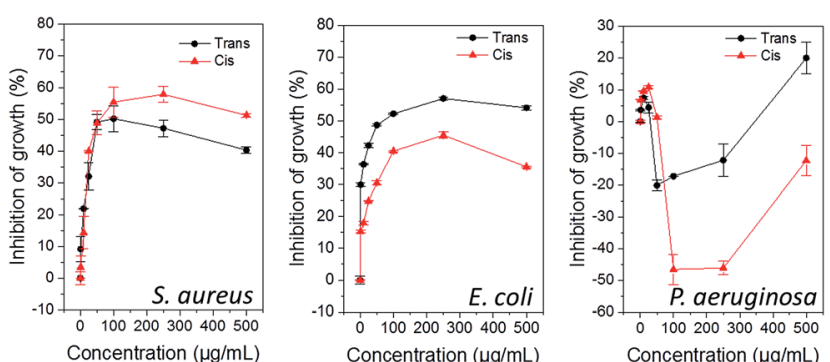

Fig. 4 Photocontrollable influence of cis- and trans-dominated photostationary states of AzoMan on bacterial activity after $24 \mathrm{~h}$. form was generally more toxic, with the exception of AzoGlcNAc, which was more toxic in its native trans form. Conversely, these compounds were typically more potent in their native trans state against E. coli, with the exception of AzoRha and AzoGlcNAc which showed higher antibacterial activity after photoisomerization. Surprisingly, all carbohydrate-based surfactants did not display significant toxicity against $P$. aeruginosa (with the exception of AzoAra) and generally promoted bacterial growth, especially in the cis photostate. Interestingly, the Lrhamnose based surfactant AzoRha was most influential in promoting growth of this bacterium. Whilst it is difficult to rationalize the heterogeneous responses observed with these amphiphiles against Gram-negative and Gram-positive bacteria, particularly those of AzoRha and AzoGlcNAc, it is interesting to note that these surfactants incorporate L-rhamnose and $N$-acetyl glucosamine as monosaccharide head groups, respectively. These carbohydrates have been known to play important roles in biofilm growth, adhesion and virulence in Gram-negative and Gram-positive species. For example, $\mathrm{N}$ acetyl glucosamine is a major constituent of the cell wall and the extracellular matrix in both Gram-positive and Gram-negative bacteria and plays important roles in virulence and biofilm formation in both groups of bacteria. ${ }^{24}$ Gram-negative bacteria, in particular Pseudomonas spp., produce surface-active rhamnolipids as virulence factors that regulate swarming motility, intercellular signalling and biofilm formation., ${ }^{4,25}$ As such, the significance of these carbohydrate head groups in bacterial evolution might be responsible for their selective mode of interaction with these bacteria. Whilst the cationic surfactant AzoTAB seems to possess a non-selective mode of antibacterial action toward these bacteria, the type of head group and the isomeric state of the tail group in carbohydrate-based surfactants are both important determinants for imparting selective growth/toxicity against different bacteria.

\section{Photocontrollable biofilm studies}

Having established the bacteria-specific influence of these surfactants on cell viability, we next sought to evaluate the effect of trans-cis photoisomerization on their ability to influence bacterial biofilm formation. Biofilms are typically formed by adherence of sessile bacteria onto a surface followed by the production of a complex extracellular matrix, thus allowing these bacterial communities to become more resistant to host immune responses and antibiotic therapies. Not only are the mechanisms of biofilm formation poorly understood, the ability to inhibit biofilm formation from pathogenic bacteria is extremely challenging. ${ }^{26}$ The intriguing effect of amphiphilic carbohydrates on biofilm growth of $P$. aeruginosa has been recently demonstrated and was shown to be heavily dependent on the length and relative configuration of the oligosaccharide head group (D-gluco or D-galacto). ${ }^{6}$ However, the influence of different photoisomers of amphiphilic carbohydrates on the biofilm forming ability of bacteria has never been reported. To understand the influence of the trans and cis isomers of these surfactants on the biofilm forming ability of bacteria, these compounds were exposed to a Gram-positive methicillin- 
resistant S. aureus (MRSA - ATCC1698) and a multi-drug resistant clinical isolate of Gram-negative $P$. aeruginosa (MDR283/1-6), both of which are known to cause problematic biofilm-associated infections., ${ }^{2,27}$

Compounds were screened for biofilm inhibition activity using a microtiter dish biofilm formation assay that involved recording the $\mathrm{OD}_{550}$ of crystal violet-treated bacterial biofilms. ${ }^{28}$ Similar to the antibacterial studies, low concentrations of surfactants showed similar effects in both cis and trans-dominated PSS, whereas the difference in activity between the photoisomeric states became more pronounced at higher concentrations (representative data for compound AzoMan is shown in Fig. 5; additional data for surfactants are shown in ESI Fig. S10 and S11 $)$. In line with the antibacterial data, these surfactants generally compromised the biofilm forming ability of Gram-positive $S$. aureus at all concentrations tested, and conversely promoted biofilm formation of Gram-negative MDR $P$. aeruginosa, particularly on exposure to higher concentrations of these compounds. In the case of $S$. aureus, AzoXyl, AzoMan, and AzoGlcNAc could inhibit bacterial biofilms more efficiently after photoexcitation, whereas the native trans states of AzoGlc, AzoRha and AzoAra remained more potent biofilm inhibitors. Similar to the antibacterial results, AzoRha revealed an interesting trend, such that lower concentrations of this compound in both the cis and trans PSS inhibited $S$. aureus biofilm formation, whereas higher concentrations of the cis isomer started promoting biofilm formation. Another notable observation was that while AzoGlcNAc displayed selective antibacterial activity against $S$. aureus in the trans form, the cisdominated photostationary state was more effective at inhibiting biofilm growth of this bacterium, suggesting a mechanism that was independent of cell death. ${ }^{29}$

\section{Bacterial motility studies}

Bacterial motility is an important physiological process that enables bacteria to seek new environments and nutrients, and as such, plays important roles in the pathogenesis as well as biofilm growth, dispersal, structure and function. ${ }^{30}$ Many bacterial species produce biosurfactants in response to environmental and internal cues, which results in a decrease in interfacial tension that enhances cellular motility and along
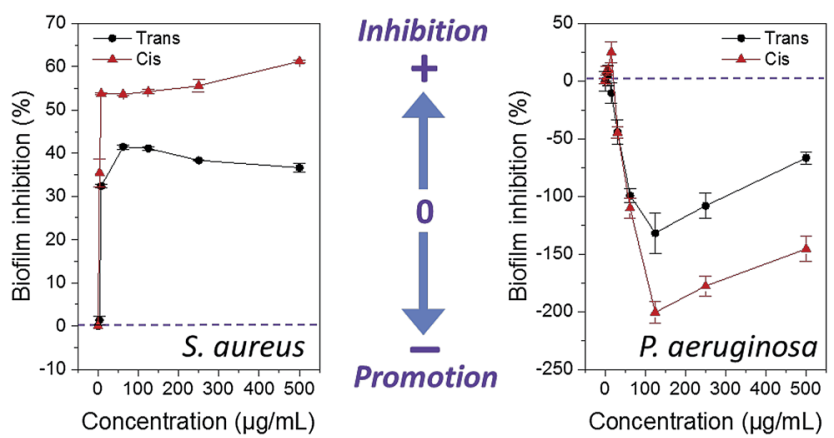

Fig. 5 Influence of cis-and trans-dominated photostationary states of AzoMan on biofilm formation of $S$. aureus and $P$. aeruginosa. surfaces and in solution. To help better understand the intriguing biofilm modulatory activity of the surfactants, we decided to probe whether these compounds were affecting bacterial motility in solution and swarming motility on a semisolid surface. We measured the dynamics and rates of swimming motility using differential dynamic microscopy (DDM) in the presence of representative AzoRha and AzoMan. DDM is a technique that uses optical microscopy to conduct an analysis similar to that used in dynamic light scattering. It has been shown that the technique is ideal for the measurement of dynamics and rates of motility of cells in bulk solution ('swimming'). ${ }^{31}$ To this end, P. aeruginosa MDR283/1-6 was chosen as the model organism as it shows sufficiently fast rates of swimming required for the timescales measured using DDM. Using this technique, these photosurfactants did not appear to significantly affect the rate of swimming in solution up to concentrations of $500 \mu \mathrm{g} \mathrm{mL} \mathrm{mL}^{-1}$, in both the cis- and transdominated PSS (ESI, Fig. S12 $\dagger$ ). Based on this observation, it is therefore unlikely that these compounds are effecting biofilm growth through changes in the swimming motility of this bacterial species.

In the case of $P$. aeruginosa, compounds were shown to weakly inhibit biofilm growth at low concentrations and strongly promote biofilm growth at higher concentrations (Fig. 5). The photoexcited cis isomers were typically more or at least equally effective over their native forms in promoting biofilms of $P$. aeruginosa. Given the important roles that rhamnolipid biosurfactants play in promoting swarming motility, we decided to investigate whether the cis and trans forms of the surfactants were affecting swarming motility of $P$. aeruginosa MDR283/1-6 via photocontrollable changes in the interfacial activity. Swarming motility of $P$. aeruginosa was assessed in $0.5 \%$ agar media in the presence of the trans and cis isomers these compounds, along with controls using a previously described swarming assay (see ESI for details $\dagger$ ). ${ }^{32}$

In the native trans form, surfactants displayed a dosedependent enhancement in swarming motility (Fig. 6). The response was insensitive to the head group employed, with all compounds being more-or-less equally effective at promoting swarming motility. However, in the cis photoisomeric state, the surfactants were generally less effective at promoting swarming motility, which may be a direct consequence of the lowered interfacial activity of the cis isomer relative to the trans isomer

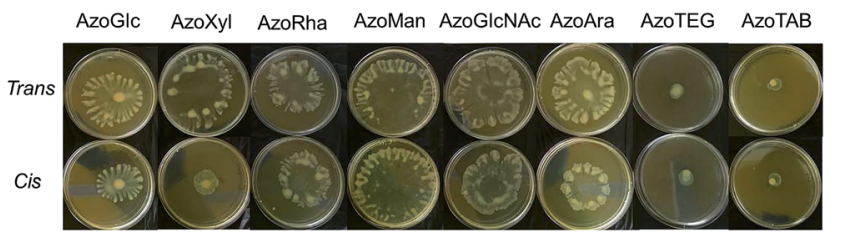

Fig. 6 Photograph of agar swarming motility assay demonstrating the influence of carbohydrate-based surfactants and control compounds in the cis- and trans-dominated PSS on the swarming motility of $P$. aeruginosa MDR283/1-6. Cells were inoculated at the centre of the agar media containing compound $\left(100 \mu \mathrm{g} \mathrm{m}^{-1}\right)$ and incubated at $30{ }^{\circ} \mathrm{C}$ for $24 \mathrm{~h}$. 


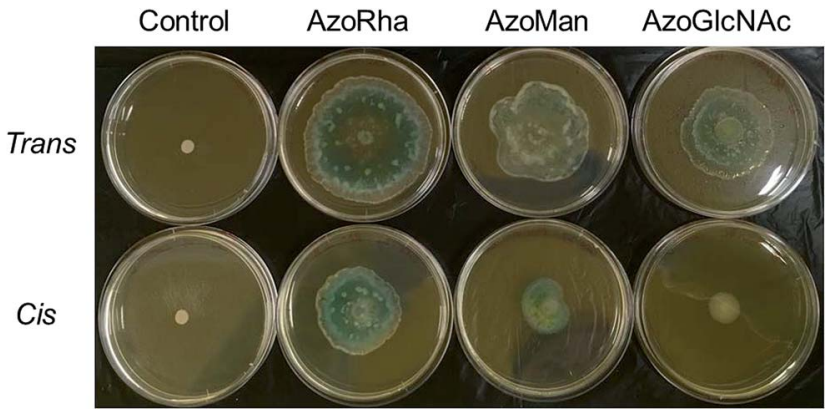

Fig. 7 Photograph of agar swarming motility assay demonstrating the influence of carbohydrate-based surfactants $\left(25 \mu \mathrm{g} \mathrm{mL}^{-1}\right)$ in the cisand trans-dominated PSS on the swarming motility of $P$. aeruginosa MDR283/1-23, a swarming negative strain.

(ESI, Fig. S13†). Interestingly, in contrast to the surfactants bearing a non-ionic carbohydrate head group, which generally promoted swarming motility, the non-surface active tail fragment AzoTEG and the cationic surfactant AzoTAB strongly inhibited swarming motility. Furthermore, unlike the carbohydrate-based surfactants, there were no significant changes in the inhibitory activity of AzoTEG and AzoTAB following trans-cis photoisomerization.

To validate the strong influence of our carbohydrate surfactants on swarming motility of $P$. aeruginosa MDR283/1-6 (a swarming-positive strain), we further investigated the influence of surfactants on the swarming motility of $P$. aeruginosa MDR283/ 1-23, a swarming-negative strain (Fig. 7). Remarkably, while untreated bacteria showed no detectable motility, these carbohydrate-based surfactants strongly promoted translocation of the bacterial colony, most likely through enhancement of surfactantmediated sliding. ${ }^{33}$ Similar to the swarming positive strain of $P$. aeruginosa, the trans configured surfactants promoted a higher level of motility relative to their cis isomers, particularly for AzoGlcNAc, which displays the largest difference in CMC between the respective trans and cis isomeric state. Taken together, these observations demonstrate the utility of UV light for modulating the swarming motility and biofilm forming ability of $P$. aeruginosa strains, which may potentially be a result of the associated changes in the interfacial activity of the cis and trans isomers of photoswitchable carbohydrate-based surfactants.

\section{Conclusions}

In summary, we report the modular synthesis and characterisation of a panel of novel carbohydrate-based surfactants and demonstrate unprecedented photo-control over bacterial growth, biofilm formation and motility using photoswitchable surfactants. Using SANS, we revealed the stark difference in the selfassembly properties of these amphiphiles, which is made possible through structural and stereochemical modification of the carbohydrate head group. Photocontrollable surface tension studies also revealed dramatic differences in the interfacial properties, which could be tuned using UV light irradiation. We then probed the impact of trans-cis azobenzene photoisomerization of these amphiphiles on their ability to modulate the growth and formation of biofilms from pathogenic, drugresistant strains of $S$. aureus and $P$. aeruginosa. Photoswitchable carbohydrate-based surfactants displayed dose-dependent, as well as bacteria- and photo-isomer specific biological activity, which suggests that these amphiphiles possess a selective mode of action. Interestingly, the photoexcited cis forms of these surfactants was shown to be more potent against $S$. aureus, while the native trans state showed higher selectivity against E. coli. Surprisingly, whilst selective antibacterial activity could be observed against $E$. coli and $S$. aureus, these compounds strongly promoted the growth of Gram-negative P. aeruginosa MDR283/16 , despite the well-documented antimicrobial activity of azobenzene dyes toward Pseudomonas spp. and other Gram-negative bacteria. Furthermore, the reduction in inhibitory potency of some surfactants at higher concentrations may suggest an antagonistic affect owing to impaired uptake of surfactant monomers and/or their metabolites due to micellization. ${ }^{34}$ Carbohydrate-based surfactants also showed remarkable bacteria-specific control over biofilm formation, such that they showed anti-biofilm activity against $S$. aureus, whereas they promoted biofilm formation in $P$. aeruginosa, with strong preference for the cis isomer. We further investigated the possible mechanisms governing the intriguing biofilm modulatory activity of these photosurfactants by measuring the rates of swimming and swarming motility of $P$. aeruginosa. Whilst the rates of swimming motility were generally insensitive to the trans and cis isomers of these compounds, surfactants were shown to strongly promote swarming motility in P. aeruginosa MDR283/1-6. We also demonstrated the ability of these compounds to promote the motility of the non-motile strain $P$. aeruginosa MDR283/1-23 on semi-solid agar, which could be further tuned using UV light. Through trans-cis photoisomerization of the azobenzene tail group, a decrease in bacterial motility could be observed relative to the trans isomer, presumably through reduction in the interfacial activity of the corresponding cis isomer. Overall, these results demonstrate the utility of UV-visible light for modulating the physicochemical properties of surfactants in order to control the physiological behaviour of bacteria. Further work is underway to understand the mechanistic interaction of the trans- and cisphotoisomeric states bearing different head groups with bacteria and their extracellular matrices, including lectin-specific inhibitors of bacterial adhesion and fluorescent uptake studies, as well as the development of dual visible light near infrared photoswitchable surfactants for in vivo applications.

\section{Acknowledgements}

B. W thanks the Australian Research Council for the Discovery Early Career research award (DE130101673). V. B. thanks Australian Research Council for a Future Fellowship (FT140101285). R. R. and W. Z. acknowledge RMIT University for Vice Chancellor's Postdoctoral Fellowship and Vice Chancellor's PhD Scholarship, respectively.

\section{Notes and references}

1 J. Carlet, et al., Lancet, 2011, 378, 369-371. 
2 J. W. Costerton, P. S. Stewart and E. P. Greenberg, Science, 1999, 284, 1318-1322.

3 (a) A. de Jesús Cortés-Sánchez, H. Hernández-Sanchez and M. E. Jaramillo-Flores, Microbiol. Res., 2013, 168, 22-32; (b) M. Inès and G. Dhouha, Carbohydr. Res., 2015, 416, 59-69.

4 J. D. Van Hamme, A. Singh and O. P. Ward, Biotechnol. Adv., 2006, 24, 604-620.

5 (a) M. Harmse, L. Yang, S. J. Pamp and T. Tolker-Nielsen, FEMS Immunol. Med. Microbiol., 2010, 59, 253-268; (b) M. E. Davey, N. C. Caiazza and G. A. O'Toole, J. Bacteriol., 2013, 185, 1027-1036.

6 E. L. Dane, A. E. Ballok, G. A. O'Toole and M. W. Grinstaff, Chem. Sci., 2014, 5, 551-557.

7 (a) I. Tochitsky, M. R. Banghart, A. Mourot, J. Z. Yao, B. Gaub, R. H. Kramer and D. Trauner, Nat. Chem., 2012, 4, 105-111; (b) M. Volgraf, P. Gorostiza, R. Numano, R. H. Kramer, E. Y. Isacoff and D. Trauner, Nat. Chem. Biol., 2006, 2, 4752; (c) A. A. Beharry and G. A. Woolley, Chem. Soc. Rev., 2011, 40, 4422-4437.

8 (a) M. Stein, S. J. Middendorp, V. Carta, E. Pejo, D. E. Raines, S. A. Forman, E. Sigel and D. Trauner, Angew. Chem., Int. Ed., 2012, 51, 10500-10504; (b) M. Schonberger and D. Trauner, Angew. Chem., Int. Ed., 2014, 53, 3264-3267; (c) W. A. Velema, J. P. van der Berg, M. J. Hansen, W. Szymanski, A. J. Driessen and B. L. Feringa, Nat. Chem., 2013, 5, 924-928; (d) C. Falenczyk, M. Shiedel, B. Karaman, T. Rumpf, N. Kuzmanovic, M. Grøtli, W. Sippl, M. Jung and B. Konig, Chem. Sci., 2014, 5, 4794-4799; (e) W. A. Velema, W. Szymanski and B. L. Feringa, J. Am. Chem. Soc., 2014, 136, 2178-2191; (f) J. Briochhagen, J. A. Frank and D. Trauner, Acc. Chem. Res., 2015, 48, 1947-1960.

9 (a) D. Bléger, J. Swarz, A. M. Brouwer and S. Hecht, J. Am. Chem. Soc., 2012, 134, 20597-20600; (b) S. Samanta, T. M. McCormick, S. K. Schmidt, D. S. Seferos and G. A. Woolley, Chem. Commun., 2013, 49, 10314-10316; (c) A. A. Beharry and G. A. Woolley, Chem. Soc. Rev., 2013, 40, 4422-4437.

10 (a) M. Dong, A. Babalhavaeji, S. Samanta, A. A. Beharry and G. A. Woolley, Acc. Chem. Res., 2015, 48, 2662-2670; (b) Y. H. Tsai, S. Essig, J. R. James, K. Lang and J. W. Chin, Nat. Chem., 2015, 7, 554-561.

11 (a) O. Srinivas, N. Mitra, A. Surolia and N. Jayaraman, J. Am. Chem. Soc., 2002, 124, 2124-2215; (b) T. Weber, V. Chandrasekaran, I. Stamer, M. B. Thygesen, A. T. Terfort and T. K. Lindhorst, Angew. Chem., Int. Ed., 2014, 53, 14583-14586.

12 J. Eastoe and A. Vesperinas, Soft Matter, 2005, 1, 338-347.

13 (a) T. Muraoka, K. Kinbara and T. Aida, Nature, 2006, 440, 512-515; (b) Y. Wang, N. Ma, Z. Wang and X. Zhang, Angew. Chem., Int. Ed., 2007, 46, 2823-2836.

14 (a) R. F. Tabor, D. D. Tan, S. S. Han, S. A. Young, Z. L. E. Seeger, M. J. Pottage, C. J. Garvey and B. L. Wilkinson, Chem.-Eur. J., 2014, 20, 13881-13884; (b) Y. Hu, J. B. Marlow, R. Ramanathan, W. Zou, H. G. Tiew, M. J. Pottage, V. Bansal, R. F. Tabor and B. L. Wilkinson, Aust. J. Chem., 2015, 68, 1880-1884.
15 R. F. Tabor, M. J. Pottage, C. J. Garvey and B. L. Wilkinson, Chem. Commun., 2015, 51, 5509-5512.

16 (a) H. C. Kolb, M. G. Finn and K. B. Sharpless, Angew. Chem., Int. Ed., 2001, 40, 2004-2021; (b) L. Bornaghi, B. L. Wilkinson, S. -A. Poulsen and T. A. Houston, Tetrahedron, 2005, 62, 8115-8125.

17 E. Chevallier, A. Mamane, H. A. Stone, C. Tribet, F. Lequeux and C. Monteux, Soft Matter, 2011, 7, 7866-7874.

18 H. Fliegl, A. Kohn, C. Hattig and R. Ahlrichs, J. Am. Chem. Soc., 2003, 125, 9821-9827.

19 M. K. Adam, J. S. Poisson, Y. Hu, G. Prasannakumar, M. J. Pottage, R. N. Ben and B. L. Wilkinson, RSC Adv., 2016, 6, 39240-39244.

20 (a) C. T. Lee Jr, K. A. Smith and T. A. Hatton, Langmuir, 2009, 25, 13784-13794; (b) A.-L. LeNy and C. T. Lee Jr, J. Am. Chem. Soc., 2006, 128, 6400-6408.

21 (a) J. A. Johnson, M. -L. Saboungi, P. Thiyagarajan, R. Csencsits and D. Meisel, J. Phys. Chem. B, 1999, 103, 5963; (b) T. Shang, K. A. Smith and T. A. Hatton, Langmuir, 2006, 22, 1436-1442.

22 J. Campbell, Current Protocols in Chemical Biology, 2010, 2, 195-208.

23 W. Paulus, in Dictionary of Microbicides for the Protection of materials, ed. W. Paulus, Springer, 1st edn, 2005, ch. 2, pp. 9-23.

24 (a) A. M. Abdel-Mawgoud, F. Lépine and E. Déziel, Appl. Microbiol. Biotechnol., 2010, 86, 1323-1336; (b) N. Cerca and K. K. Jefferson, FEMS Microbiol. Lett., 2008, 283, 36-41; (c) A. Kropec, T. Maira-Litran, K. K. Jefferson, M. Grout, S. E. Cramton, F. Götz, D. A. Goldmann and G. B. Pier, Infect. Immun., 2005, 73, 6868-6876.

25 (a) S. J. Pamp and T. Tolker-Nielsen, J. Bacteriol., 2007, 189, 2531-2539; (b) N. C. Caiazza, R. M. Q. Shanks and T. A. O'Toole, J. Bacteriol., 2005, 187, 7351-7361.

26 T. F. Mah, B. Pitts, B. Pellock, G. C. Walker, P. S. Stewart and G. A. O'Toole, Nature, 2003, 426, 306-310.

27 T. Bjarnsholt, APMIS, 2013, 121, 1-58.

28 G. A. O'Toole, J. Visualized Exp., 2011, 47, DOI: 10.3791/2437. 29 J. L. Lister and A. R. Horswill, Front. Cell. Infect. Microbiol., 2014, 4, 1-9.

30 (a) G. A. O'Toole and R. Kolter, Mol. Microbiol., 1998, 30, 295304; (b) N. Verstraeten, K. Braeken, B. Debkumari, M. Fauvart, J. Fransaer, J. Vermant and J. Michiels, Trends Microbiol., 2008, 16, 496-506.

31 (a) L. G. Wilson, V. A. Martinez, J. Schwarz-Linek, J. Tailleur, G. Bryant, P. N. Pusey and W. C. K. Poon, Phys. Rev. Lett., 2011, 106, 18101-18104; (b) V. A. Martinez, R. Besseling, O. A. Croze, J. Tailleur, M. Reufer, J. Scwarz-Linek, L. G. Wilson, M. A. Bees and W. C. K. Poon, Biophys. J., 2012, 103, 1637-1647.

32 T. Inoue, R. Shingaki and K. Fukui, FEMS Microbiol. Lett., 2008, 281, 81-86.

33 D. B. Kearns, Nat. Rev. Microbiol., 2010, 8, 633-644.

34 (a) S. Piotto, S. Concilio, L. Sessa, A. Porta, E. C. Calabrese, A. Zanfardino, M. Varcamonti and P. Iannelli, Eur. J. Med. Chem., 2013, 68, 178-184; (b) M. Hartman, H. Papavlassopoulos, V. Chandrasekaran, C. Grabosch, F. Beiroth, T. Lindhorst and C. Röhl, FEBS Lett., 2012, 586, 1459-1465. 